\title{
10. \\ Sul proemio degli Anekdota di Procopio
}

\author{
Giovanni Parmeggiani *
}

\author{
DOI: http://dx.doi.org/10.7359/820-2017-parm
}

ABSTRACT: This paper offers a new analysis of the proem of Procopius' Anekdota (I 1-10). The author discloses Procopius' own original rework of, and polished intertextual dialogue with, Greek historians of the $\mathrm{V}$ and the IV century BC such as Thucydides and Theopompus (An. I 2-3 Theop. FGrHist 115 T 20a and T 20a'; An. I 4-5 Thuc. I 21.1), thus showing that the proem of the Anekdota is not a mere collection of topoi. While introducing the Anekdota to his readers, Procopius appears to be self-aware of the fundamental tasks of historical inquiry. He presents his work as a piece of real historical inquiry, and specifically, as an aetiological supplement to the Bella.

KEYWORDS: Historical and historiographical causes, Procopius, proem, Theopompus, Thucydides - Cause storiche e storiografiche, Procopio, proemio, Teopompo, Tucidide.

La reale natura degli Anekdota e la loro pertinenza effettiva al genere storiografico sono punti dubbi, sembrerebbe, fin dall'antichità. Il lessico della Suda, alla voce Prokopios, definisce gli Anekdota come un concentrato di psogoi e come una komodia ai danni di Giustiniano, Teodora, Belisario e la moglie di questi:

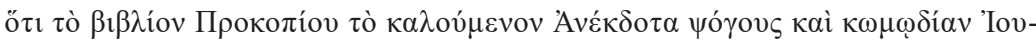

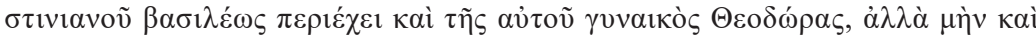

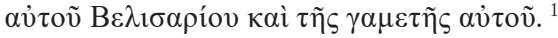

Non è una prospettiva così distante da quella di quanti, tra i moderni, hanno giudicato gli Anekdota non più di un velenosissimo pamphlet, un libello segnato da quell'acredine da cui ogni sana e autentica inchiesta storica non dovrebbe mai, in linea di principio, restare intaccata ${ }^{2}$. Se però è lecito,

* Desidero ringraziare gli organizzatori del convegno per di avermi dato modo di rendere, con questo contributo, un piccolo omaggio alla cara memoria di Antonio Baldini.

1 Suid. $\pi 2479$ Adler, s.v. Проко́ $九$ เos. Si ricordi anche la definizione di «palinodia» data da Niceforo Callisto Xantopulo (HE XVII 10). Sulla ricezione antica degli Anekdota, vd. Cameron 1985, 47-48.

2 Vd. ad esempio Cameron 1985, 47 ss. C'è chi non ha esitato a liquidare gli Anekdota come una brillante fiction, di fatto inutile alla ricostruzione degli avvenimenti reali (Brubaker 
tentando definizioni, guardare alla storia della ricezione, appare comunque fondamentale cercare anche di capire come Procopio stesso concepisse gli Anekdota. A questo scopo, qui esamineremo il luogo in cui logicamente ci si aspetterebbe di trovare qualche indizio utile, il proemio dell'opera.

Leggiamo in esordio al proemio degli Anekdota (I 1-2):

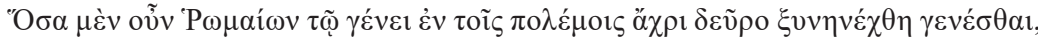

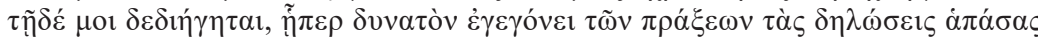

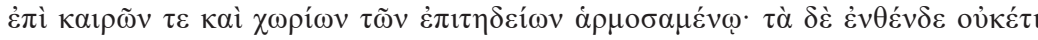

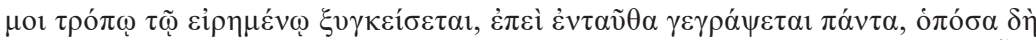

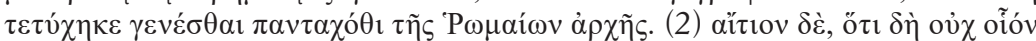

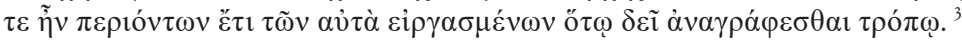

Colpiscono l'assenza del sigillo e il parallelismo lessicale con un altro proemio, quello del libro VIII - e ultimo - dei Bella. Infatti, nel proemio del libro VIII dei Bella Procopio ricorda come la pubblicazione già avvenuta dei precedenti sette libri renda impossibile aggiungere notizie al materiale già scritto, in quanto già pubblicato, e ciò lo costringa a cambiare la modalità di arrangiamento (o tropos) dell'esposizione: da questo momento in poi dichiara Procopio - le vicende accadute non saranno più esposte secondo i luoghi del loro svolgimento ${ }^{4}$. In esordio al proemio degli Anekdota, pure, Procopio asserisce di essere costretto a cambiare il tropos dell'esposizione e di non poter più raccontare gli eventi secondo i luoghi in cui si svolsero, ma, questa volta, perché intende raccontare gli eventi in forma completa: più precisamente, Procopio dichiara di voler raccontare cose che

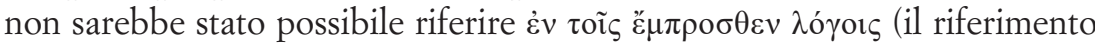

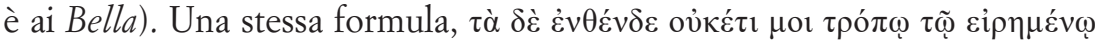

2004, 101); c'è chi ne ha evidenziato, all'opposto, il senso di un supplemento ai Bella di fondamentale valore storico (in particolare Kaldellis 2004, 45 ss.), o ancora di una replica alla propaganda di Giustiniano, quand'anche iperbolica, comunque fondatamente critica (e.g. Greatrex 2014a, 100-101). Altri, infine, ne hanno ribadito la pertinenza al genere dell'invettiva, ritenendo gli Anekdota un'opera composta come autodifesa contro possibili, venturi delatores quando, morta Teodora (548), la fine dell'impero di Giustiniano sembrava imminente (così, ultimamente, Börm 2015, sulla scia di una tesi formulata da Signes Codoñer). Sulle tesi moderne circa la natura e la funzione degli Anekdota, vd. ora Greatrex 2014a; Greatrex 2014b, 121c; Börm 2015 (con bibliografia).

3 Proc. An. I 1-2, III, p. 4 Haury-Wirth.

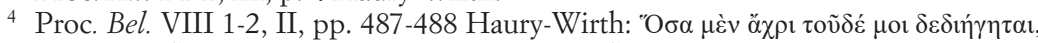

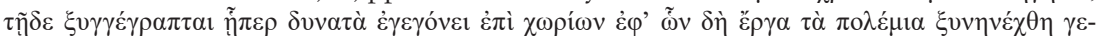

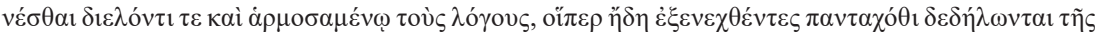

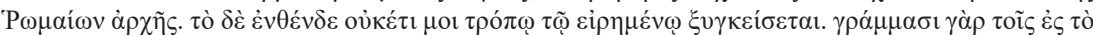

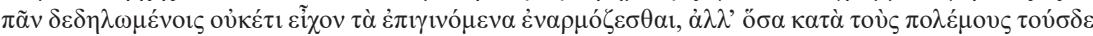

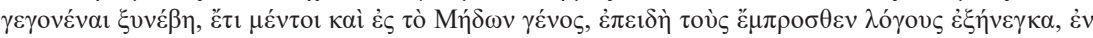

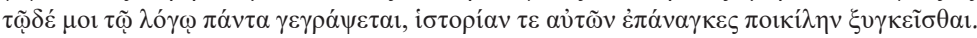




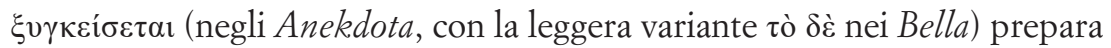
due risoluzioni differenti. Eppure, complici anche le altre corrispondenze lessicali ben visibili, disseminate nei rispettivi contesti ${ }^{5}$, il parallelismo tra i due passi resta, e talmente stretto da non poter passare inosservato al lettore: questi doveva capire che gli Anekdota erano in continuità reale con i Bella ${ }^{6}$; e che gli Anekdota, pur sprovvisti di sigillo, erano anch'essi dello stesso autore dei Bella, Procopio appunto ${ }^{7}$.

Procopio si rende dunque visibile senza dichiararsi. La formula $\tau \grave{\alpha} \delta \grave{\varepsilon}$

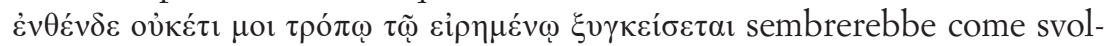
gere la funzione di un sigillo - un implicito sigillo: l'opera segreta di Procopio si apre così, all'insegna della segretezza.

L'autore, si diceva, intende raccontare negli Anekdota cose che non sarebbe stato possibile riferire prima: vuole scrivere «tutto quanto è accaduto in ogni luogo dell'impero romano», fornire un resoconto completo degli

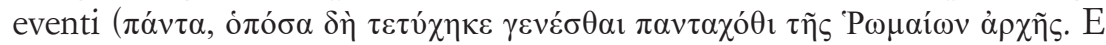
qui è notevole l'insistenza sul concetto di totalità: $\pi \alpha ́ v \tau \alpha \ldots \pi \alpha v \tau \alpha \chi \theta_{0} \theta$ ). Egli chiaramente allude ai Bella - o meglio, a quanto dei Bella risultava già pubblicato al tempo della composizione degli Anekdota ${ }^{8}$ - come a un'opera parziale, segnata dall'incompletezza. Per contro, egli annuncia gli Anekdota come un'opera integrativa. Ora, quale sia esattamente la natura e l'entità dell'incompletezza dei Bella, ovvero il contributo reale dell'integrazione che si vuole portare con gli Anekdota, viene chiarito da Procopio nel seguito (I 2-3):

5 Sulle analogie tra l'esordio del proemio degli Anekdota e l'esordio del libro VIII dei Bella, vd. Rubin 1957, 533.

6 Non ha torto Leppin - Meier 2005, 283, nel sostenere che la prefazione agli Anekdota abbia la funzione di sottolineare il passaggio dai Bella agli Anekdota. Ma ciò non ne diminuisce, a mio avviso, l'identità di proemio a tutti gli effetti (vd. infra).

7 Osserva ragionevolmente Kaldellis 2010, xxvii-xxviii: «The beginning of the preface to Book 8 [...] copies the beginning of the preface of The Secret History, indicating the continuity of Prokopios' intention and providing an inside joke to those in his inner circle of friends who had read the secret work». Credo però che Procopio guardasse anche ai lettori futuri: il codice chiarirà l'identità dell'autore dell'opera anche a chi, come viene a precisarsi più avanti nel proemio, si troverà nella condizione di poter vagliare l'attendibilità del contenuto degli Anekdota alla luce delle testimonianze coeve (I 5, III, p. 5

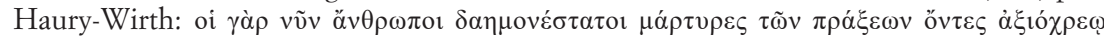

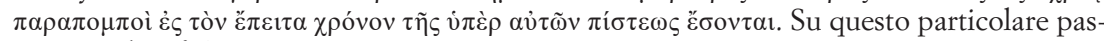
saggio, vd. infra).

8 Convenzionalmente si ritiene che gli Anekdota siano stati completati intorno al 550, il libro VIII dei Bella nel 554 ca. Vd. Cameron 1985, 52-53; Kaldellis 2004, 46. Croke 2005 ha rilanciato la possibilità che gli Anekdota siano stati ultimati dopo la realizzazione del libro VIII dei Bella, e precisamente nel 558-559; per una replica a questa posizione, Kaldellis 2009. 


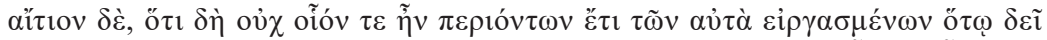

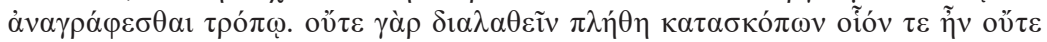

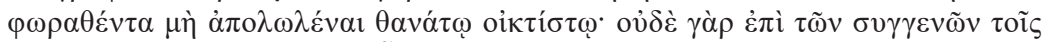

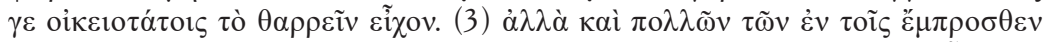

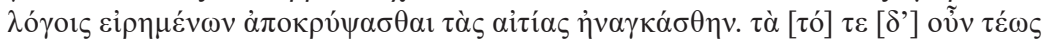

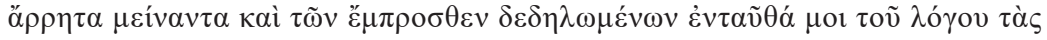

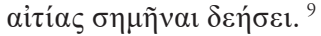

Qui l'autore avverte il lettore, con dovizia di particolari, di essere stato costretto, componendo i Bella, a delle omissioni, e questo perché la natura compromettente di certe informazioni, se fornite, avrebbe messo a repentaglio la sua vita. I potenti che al tempo erano ancora viventi, evidentemente i referenti delle informazioni in questione (e noi sappiamo di chi si tratta: Giustiniano, Teodora, Belisario) non avrebbero certo tollerato la circolazione di simili compromettenti notizie, e Procopio - si noti - descrive tutti costoro non identificandoli col nome e nel loro ruolo ufficiale, bensì con la

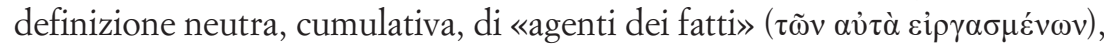
ribadendo così la loro centralità nell'accadimento degli eventi. Non a caso Procopio specifica che le omissioni avrebbero riguardato proprio il piano

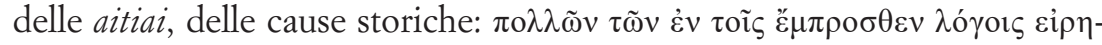

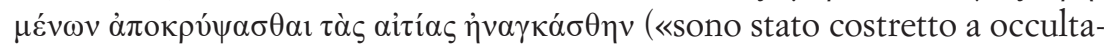
re le ragioni di molte cose dette negli scritti precedenti [cioè nei Bella]»). Il

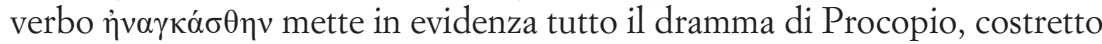
dalle circostanze a secretare, componendo i Bella, le cause delle azioni sto-

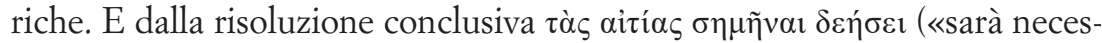
sario indicare le cause»), che riprende l'osservazione iniziale ov̉ $\chi$ oíóv ... ö $\tau \omega$

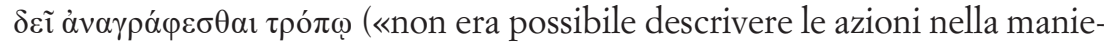
ra necessaria») si capisce che Procopio vuole compensare, con gli Anekdo$t a$, il grave difetto eziologico dei Bella sentendo questo effettivamente come un proprio compito essenziale, come un proprio dovere irrinunciabile.

Procopio, dunque, fa ammenda. È consapevole di essere venuto meno, nei Bella, a un compito primario dello storico di professione, quello di dichiarare le cause. Chi è storico di professione, in effetti, non dovrebbe mai, in linea di principio, occultare le cause (à dovrebbe procedere in senso esattamente opposto, rivelare le cause invisibili. E questo è quel «dovere» che Dionigi di Alicarnasso descriveva come caratteristica fondamentale dell'inchiesta di Teopompo di Chio, storico del IV secolo a.C., nella Lettera a Pompeo Gemino, 6 (FGrHist 115 T 20a), «per ogni fatto non solo vedere e dire le cose visibili ai più, ma indagare sia le ragioni invisibili dei fatti e di chi li ha compiuti, sia i sentimenti dell'a-

9 Proc. An. I 2-3, III, p. 4 Haury-Wirth. 
nima, che non sono facili a conoscersi per i più; e rivelare tutti i misteri sia della virtù apparente sia della sconosciuta malvagità»:

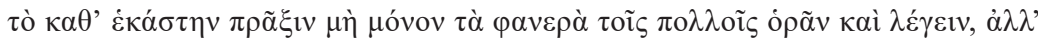

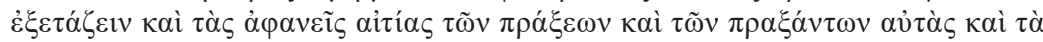

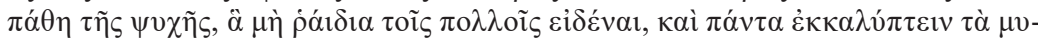

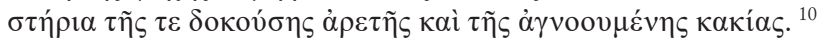

Un dovere strettamente connesso alla parresia, la libertà di giudizio e di parola che si manifesta nel «non occultare le cause irrivelate sia dei fatti sia dei discorsi», come ci ricorda l'epitomatore del perduto trattato di Dionigi di Alicarnasso Sull'imitazione, ancora in riferimento alle virtù di Teopompo, in un luogo chiaramente parallelo a quello, già citato, della Lettera a Pompeo Gemino:

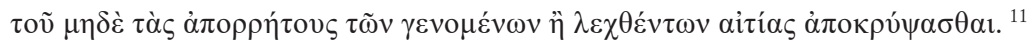

Vi sono ben pochi dubbi che Procopio, nel punto del proemio degli Anekdota in cui riflette sulle cause e ammette di essere venuto meno al dovere di

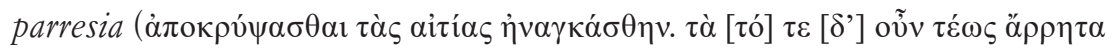

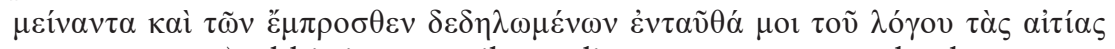

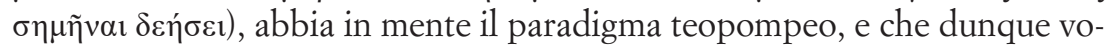
glia dare agli Anekdota il senso di una rivelazione eziologica, di uno sguardo più profondo sulle azioni e sugli agenti delle stesse, quale può vantare unicamente chi sia in confidenza con i potenti in quanto protagonisti e motori degli eventi (e in effetti, non sarebbe ardito sostenere che Procopio vantasse con la corte di Giustiniano una confidenza almeno simile a quella di Teopompo con la corte di Filippo II) ${ }^{12}$.

10 Dion. Hal. Ad Pomp. 6.7, VI, p. 246, 10-16 U-R = Theop. FGrHist 115 T 20a. Sul passo teopompeo, vd. ora Vattuone 2014.

11 Dion. Hal. De imit. 3.3, VI, p. 209, 17-20 U-R = Theop. FGrHist 115 T 20a ${ }^{+}$.

12 Già Hirzel 1892, 370-374, individuava in Teopompo autore dei Philippika, in ragione di T 20a, l'artefice di una Geheimgeschichte di cui gli Anekdota di Procopio sarebbero eredi. E in Teopompo si è a lungo riconosciuto l'archetipo di una storiografia votata allo psogos di cui anche Procopio, a sua volta, sarebbe esponente significativo appunto negli Anekdota (una visione, questa, a dir poco riduttiva e discutibile non soltanto per quanto concerne la storiografia di Teopompo [vd. Vattuone 2014, e sull'inconsistenza del concetto di «storiografia retorica», ora, Parmeggiani 2011, 34 ss., in partic. 47 ss. su lode e biasimo], ma anche per quanto riguarda la storiografia di Procopio [vd. qui infra]). Né Hirzel 1892 - se si fa eccezione per l'accenno alquanto vago di 372 con n. 3 - né altri, a quanto mi risulta, hanno comunque colto l'eco teopompea diretta e specifica che io credo si possa individuare qui nel proemio degli Anekdota. Studi particolari sul proemio in questione hanno semmai segnalato - non in questo preciso punto, ma più avanti nel testo - echi polibiani e diodorei (Lieberich 1900, 2-3; Rubin 1957, 533-534; cf. Cameron 1985, 48). 
E come la storiografia di Teopompo trovava il suo fulcro nella rivelazione delle cause invisibili e soltanto di qui agganciava il problema dell'ethos e della paideia dei personaggi - il problema ingiustamente scambiato, da molta critica moderna, come l'essenza dell'inchiesta teopompea - così Procopio, consapevolmente, identifica gli Anekdota come un progetto eminentemente eziologico: il giudizio sull'ethos dei personaggi e sulla loro paideia, in realtà, è soltanto indotto; esso è una conseguenza dell'analisi delle cause: senza questa, quello non sussiste ${ }^{13}$.

La rivelazione delle aitiai, delle cause, è dunque indicata da Procopio come la ragione primaria della composizione degli Anekdota. L'evidente volontà dell'autore di rispettare l'etica storiografica della rivelazione delle aitiai finisce per arricchire l'opera - questo «progetto eminentemente eziologico», come l'abbiamo definito - di un senso ulteriore: gli Anekdota sono visti da Procopio non soltanto come un'imprescindibile integrazione o supplemento ai Bella, ma, almeno per concezione, come una necessaria correzione di prospettiva e revisione (revisione che non comporta necessariamente, di per sé, una radicale ritrattazione) ${ }^{14}$.

Dopo aver definito la natura degli Anekdota - una natura eziologica, come si è detto -, Procopio in I 4-5 si interroga, come già tanti altri autori prima di lui, sul destino spettante all'opera:

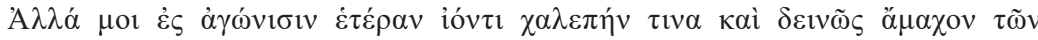

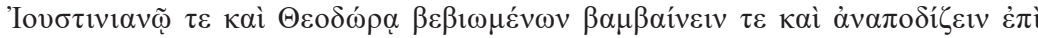

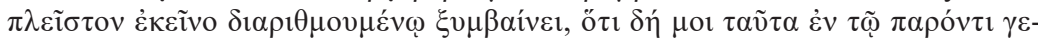

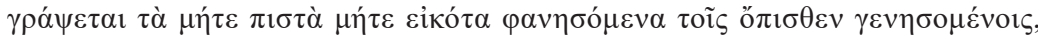

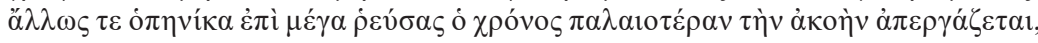

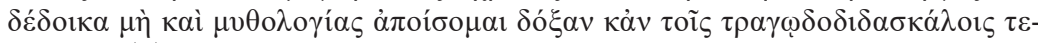

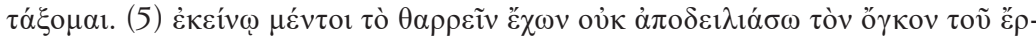

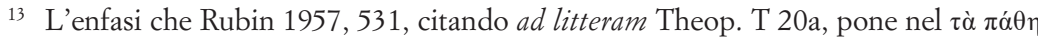
$\tau \tilde{\eta} \varsigma \psi v \chi \tilde{\eta} \varsigma$ (cf. già Hirzel 1892, 372) è indicativa di un equivoco che tocca tanto il luogo teopompeo quanto l'intento proemiale di Procopio: si doveva semmai evidenziare, nel luogo

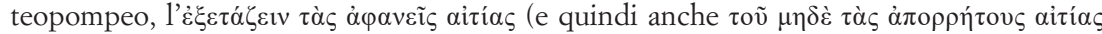

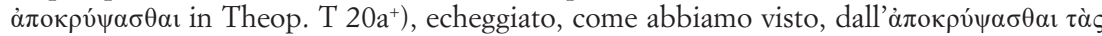

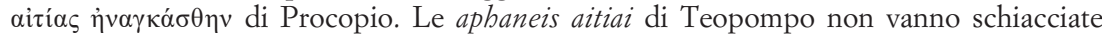
esclusivamente sui pathe.

${ }_{14}$ Quanto osservo, in fondo, avalla la tesi di Kaldellis 2004, 45 ss. (cf. anche Kaldellis 2010, xxvi-xxvii), secondo cui gli Anekdota costituirebbero un «esoteric supplement» (e qui io preferirei parlare di un supplemento eziologico) ai Bella, e non il loro rovesciamento/negazione. Trovo significativo, a questo proposito, che la Suda conti gli Anekdota insieme ai libri dei Bella, quasi costituissero una totalità e non due opere da contrapporsi

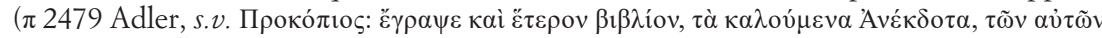

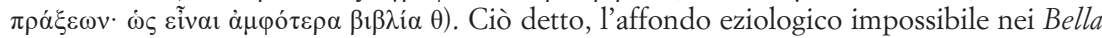
e realizzato negli Anekdota non può non incidere sulla rappresentazione/restituzione degli stessi eventi: di qui un certo margine di revisione, per certi versi obbligato. 


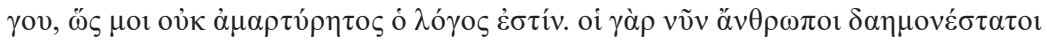

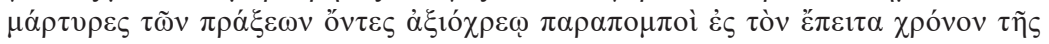

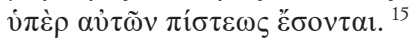

Egli, finalmente, fa il nome dei sin qui innominati «agenti dei fatti» (il $\tau \tilde{\omega} v$

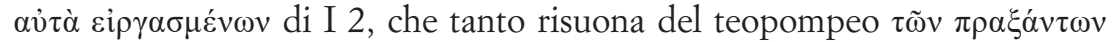

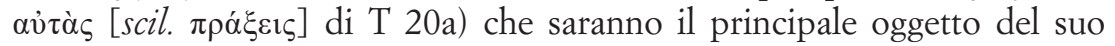
esame eziologico: Giustiniano e Teodora. La coppia imperiale viene rappresentata come in un'immobilità statuaria di corte, temibilissima, a cui Procopio si accosta con la sua parola rivelatrice non certo per ossequiare, ma con la stessa paura di un dignitario che sa di essere punibile per la sua

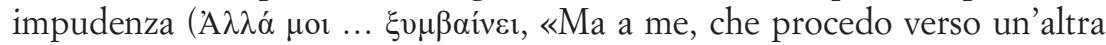
prova, difficile e terribilmente complicata da affrontare, e passo in rassegna gli eventi della vita di Giustiniano e Teodora, accade di balbettare e di tornare sui miei passi»). Ma la preoccupazione di Procopio ha una ragione più profonda della prevedibile ritorsione dell'autorità imperiale e del suo pur temibile giudizio - in fin dei conti, l'una e l'altro sono subordinati al tempo -: Procopio teme, piuttosto, per il modo in cui la sua opera sarà recepita dalle generazioni future; teme il giudizio e la condanna dei posteri. Più precisamente, Procopio è preoccupato del fatto che il suo resoconto, riguardando cose di per sé inimmaginabili, non credibili e non verosimili, una volta acquisita una «patina antica» in ragione del fluire del tempo, finisca per essere etichettato come mythologhia, e di conseguenza, che lui stesso, in quanto autore di un'opera giudicata e condannata come mythologhia, venga

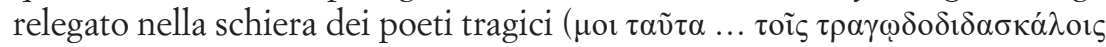
$\tau \varepsilon \tau \alpha ́ \xi o \mu \alpha l$, «ora saranno scritte da me cose che parranno incredibili e inverosimili a quelli che saranno, e nel momento in cui il tempo, scorrendo, avrà reso più antica la mia voce, ho paura che mi guadagnerò fama di mythologhia e che sarò contemplato tra i poeti tragici»). Si potrebbe pensare a Polibio come referente implicito di tale affermazione, ricordando, ad esempio, la critica che lo storico di Megalopoli formula in II 56 contro Filarco (FGrHist $81 \mathrm{~T}$ 3), sottolineando come la storia, a differenza della tragedia, non debba puntare all'ekplexis del lettore attraverso il racconto di cose terribili e favolose. Ma c'è un referente più antico e più profondo di Polibio, per questo luogo di Procopio; un referente problematico e per certi versi insospettabile: Tucidide, I 21.1, il luogo in cui lo storico ateniese attacca poeti e logografi sottolineando come l'esercizio mancato dell'elenchos com-

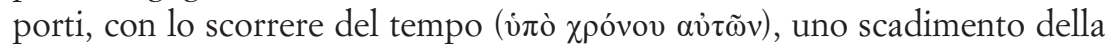

15 Proc. An. I 4-5, III, p. 5 Haury-Wirtz. 
tradizione, appunto perché «non verificata» ( $\dot{\alpha} v \varepsilon \xi \dot{\varepsilon} \lambda \varepsilon \gamma \kappa \tau \alpha)$, a «ciò che ha il

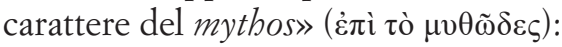

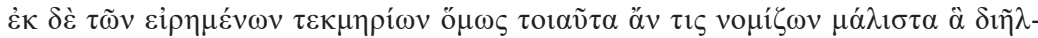

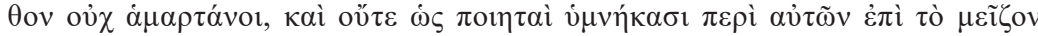

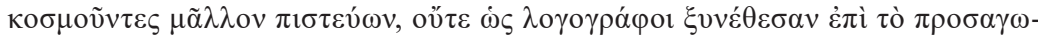

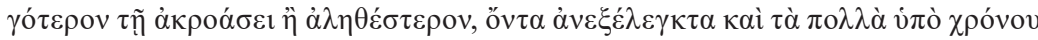

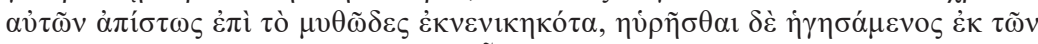

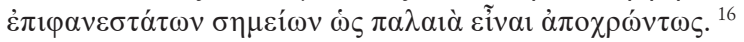

Procopio rielabora consapevolmente Tucidide (e questo non dovrebbe stupire, se si considera l'evidenza di tanti recuperi tucididei già nei Bella: Procopio vantava una grande confidenza con Tucidide ${ }^{17}$ ).

Per comprendere il senso dell'operazione compiuta da Procopio - la funzione reale di questa rielaborazione tucididea - va osservato quanto segue. Tucidide, in I 21.1, riflette sui fatti del passato e sui rischi che comporta un approccio acritico all'informazione tràdita, vale a dire, la trasmissione delle notizie ricevute senza procedere ad alcun vaglio; in questo contesto, attacca i poeti e i logografi (scrittori di poesia e di prosa). Procopio recupera la critica tucididea orientandola contro se stesso, facendo del processo descritto da Tucidide (cioè lo scadimento della tradizione, perché «non verificata», a «ciò che ha il carattere del mythos») un rischio concreto, in prospettiva, per la propria opera, in quanto essa viene a riguardare fatti di per sé incredibili e inverosimili. In altre parole, Procopio pone la propria opera sullo stesso livello delle notizie tràdite passibili di vaglio - e non a caso, egli la definisce un'akoe (I 4). Qui ci sono gli estremi di una rivoluzione: Procopio, in questo preciso punto del proemio degli Anekdota, parla della propria opera non come inchiesta e scrittura, ma come una testimonianza diretta passibile di vaglio. Ora, innanzi al problema dell'incredulità dei lettori futuri, Procopio ha la sua soluzione, che è anche quanto lo incoraggia lui dice - a procedere nella scrittura, e a sostenere di slancio la difficile pro-

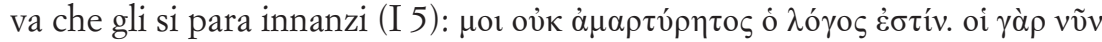

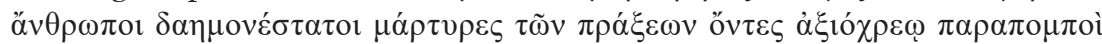

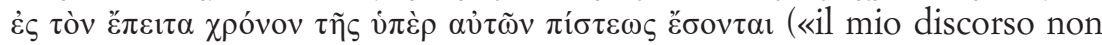
è senza testimoni. Gli uomini di ora, testimoni ben informati dei fatti in

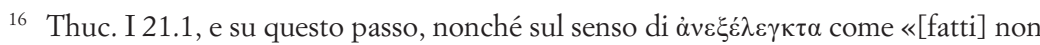
verificati», vd. Parmeggiani 2003.

17 Sul rapporto Tucidide-Procopio vd. già Braun 1885, in cui tuttavia non vi è cenno al nesso tra il proemio degli Anekdota e Thuc. I 21.1 che qui segnaliamo. Idem si dica per i principali studi sul proemio degli Anekdota (Lieberich 1900, 2-3; Rubin 1957, 533-534; cf. Cameron 1985, 48). In generale su Procopio e la storiografia classica (Tucidide in particolare), vd. Kaldellis 2004, 9 ss. e 17 ss. 
questione, saranno garanti, per il futuro, di credibilità»). Procopio tiene a sottolineare che la sua akoe - la sua testimonianza - non resterà senza va-

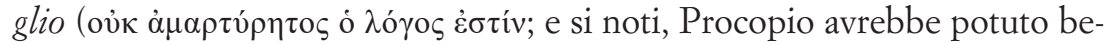

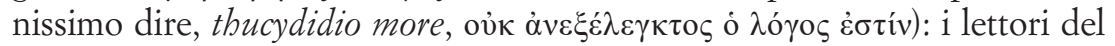
futuro che, scettici innanzi agli incredibili fatti narrati, vorranno sottoporre a verifica ciò che troveranno scritto da Procopio negli Anekdota, potranno farlo; e giungeranno, così, alla conclusione che Procopio, nonostante l'apparente inverosimiglianza delle cose raccontate, dice la verità.

Procopio, nel proemio degli Anekdota, intrattiene dunque con Tucidide, I 21.1 un raffinato dialogo intertestuale. Il racconto degli Anekdota sarà centrato sul contrasto radicale tra il phaneron e l'aphanes, l'apparenza magnifica da una parte e l'infima realtà, dall'altra, della corte imperiale; Procopio lo sa, e sa anche che il contrasto riuscirà così sconvolgente da suscitare scetticismo nel lettore. Ebbene, Procopio recupera Tucidide, I 21.1 intenzionalmente per sottoporre se stesso al più duro ed esemplare degli esami - un «auto-vaglio», una prova autentica di veridicità. Prendendo atto della natura ben particolare del suo racconto, egli delega intenzionalmente al lettore quel controllo incrociato che, in linea di principio, spetterebbe allo storico in quanto autore dell'inchiesta; così facendo, Procopio sceglie di smettere i panni di storico, e di indossare quelli del testimone passibile di verifica, apposta per ribadire la veridicità del suo racconto: gli Anekdota, questo esame eziologico finalizzato a rivelare il volto segreto degli agenti storici e delle loro azioni, sono una testimonianza diretta la cui attendibilità resisterà alla prova del tempo.

Ovviamente, il ruolo di «testimone», che Procopio ha stabilito di assumere qui, non gli preclude di indossare poi nuovamente quello di «storico» a pieno titolo. Questo avviene nell'ultima parte del proemio, là dove Procopio, sulle tracce di molta storiografica greca di età ellenistica, recupera l'idea dell'utilità del racconto del passato come modello per un corretto comportamento politico ed etico nel futuro (I 6-10):

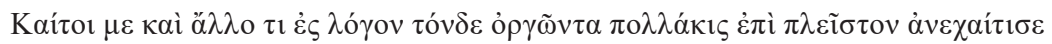

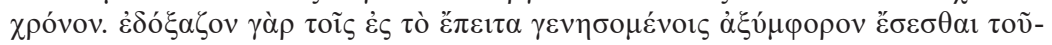

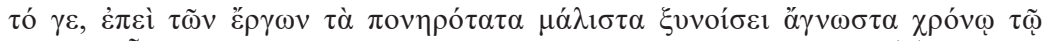

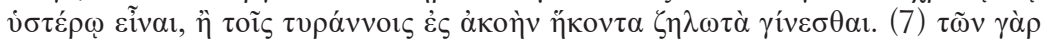

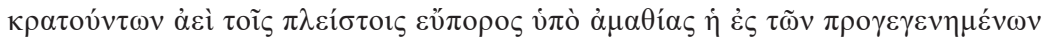

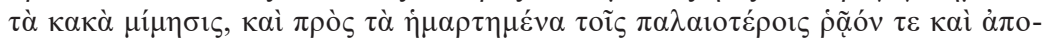

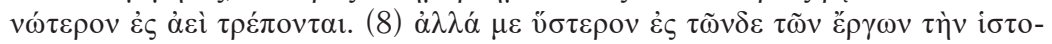

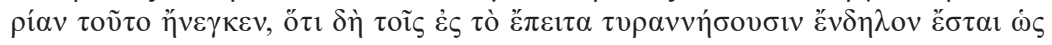

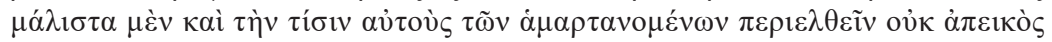

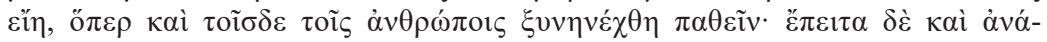

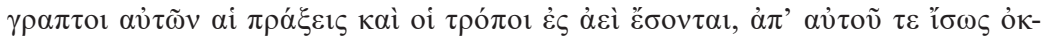

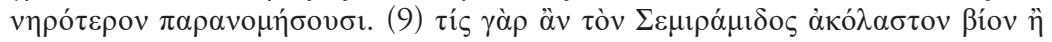




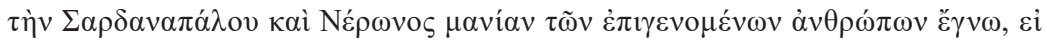

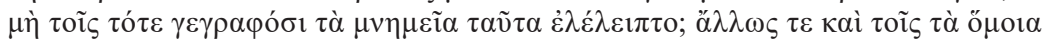

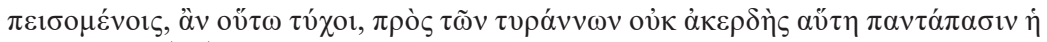

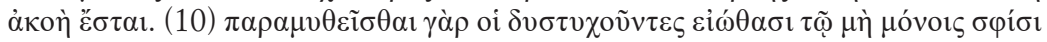

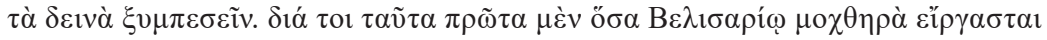

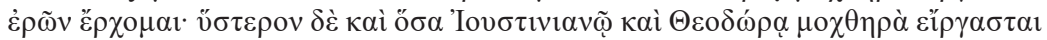
$\dot{\varepsilon} \gamma \omega \grave{\delta} \delta \eta \hat{\omega} \sigma \omega{ }^{18}$

Leggiamo in particolare in I 8, «questo mi ha spinto all'iotopía di tali eventi, l'idea che sarà chiaro, a quanti in futuro saranno tiranni, che non è improbabile che essi siano puniti per le loro azioni malvagie». Quella che prima, per scelta dell'autore, era stata akoe, ora è tornata ad essere, a esame superato, historia a pieno titolo. Ora Procopio, ergendosi a testimone $e$ storico, può ritornare a dominare tutto il tempo dall'alto e a giudicare $\mathrm{i}$ protagonisti della storia e il loro operato: non soltanto può permettersi di chiamare i primi per nome (Giustiniano, Teodora, Belisario), senza alcun timore di confronto; può anche qualificarli - e qui è la sentenza definitiva dell'inchiesta storica - come «tiranni» (tyrannoi) e definire «malefatte» le loro azioni $(\mu \mathrm{o} \chi \eta \eta \rho \grave{\alpha})^{19}$.

Una piccola riflessione conclusiva, a questo punto, è doverosa. L'analisi del proemio ha posto in evidenza, in primo luogo, come Procopio, in qualità di autore, concepisca l'opera degli Anekdota: dal suo punto di vista - ed è un dato che il lettore moderno non dovrebbe dimenticare - si tratta di un affondo eziologico, implicante una correzione di prospettiva rispetto ai Bella, non necessariamente una ritrattazione integrale. In secondo luogo, l'esame del proemio ha rivelato la presenza di un solidissimo retroterra storiografico (si sono individuati riferimenti a Tucidide e a Teopompo, in particolare). E qui è bene sottolineare come gli storiografi di $\mathrm{V}$ e IV secolo a.C. non risultino recuperati nel proemio degli Anekdota in modo superficiale, replicati in modo meccanico, quasi si trattasse di formule fisse e stereotipiche ${ }^{20}$; al contrario, sono stati utilizzati come veri interlocutori, in un dialogo intertestuale molto fine, più sottile rispetto a quello

18 Proc. An. I 6-10, III, pp. 5-6 Haury-Wirtz. Sul recupero del paradigma polibiano (la scrittura storica dà consolazione nella sofferenza: Polyb. I 1.2) e diodoreo (la scrittura storica impartisce lezioni anche ai malvagi, distogliendoli dalle cattiverie: Diod. I 1.5), vd. Lieberich 1900, 3; Rubin 1957, 534; Cameron 1985, 48.

19 Si noti il ritorno di akoe in An. I 9, III, p. 6 Haury-Wirtz, questa volta a qualificare, in modo non privo di una certa efficacia teatrale, una voce di protesta contro le

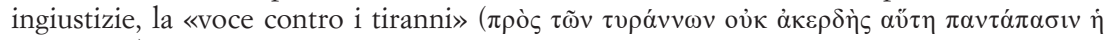

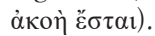

20 Non nego che nel proemio degli Anekdota vi siano topoi (vd. Leppin - Meier 2005, 283 , con riferimento alla tradizione della storiografia di età imperiale). La presente analisi però dimostra che esso non è affatto riducibile a mero collettore di luoghi comuni storiografici. 
che si conosce, ad esempio, dal proemio generale dei Bella. Procopio scrive il proemio degli Anekdota nel pieno della sua maturità storiografica, dimostrando di avere chiara coscienza della tradizione e dei doveri fondamentali del ghenos historikon; i dialoghi con Teopompo e Tucidide sono le tappe di una volontaria autocritica, funzionale a convincere il lettore circa l'assoluta serietà di intenti: Procopio vuole che il lettore non accolga lo sconvolgente contenuto degli Anekdota come il frutto di un fantasioso estro o di uno sfogo insensato, bensì come il tentativo consapevole di comprendere in profondità gli avvenimenti e di restituire questa profondità in forma integrale.

Questo forse non basterà a risolvere in via definitiva i dubbi, da sempre esistiti, sulla reale natura degli Anekdota e sulla loro pertinenza effettiva al genere storiografico; ma almeno, potrebbe dissuadere dalla tentazione di liquidarli semplicemente come il livido psogos di uno storico dimentico del suo mestiere.

\section{BIBLIOGRAFIA}

Börm 2015

Braun 1885

Brubaker 2004

Cameron 1985

Croke 2005

Greatrex 2014a

Greatrex 2014b

Hirzel 1892

Kaldellis 2004

H. Börm, Procopius, His Predecessors, and the Genesis of the «Anecdota»: Antimonarchic Discourse in Late Antique Historiography, in H. Börm (ed.), Antimonarchic Discourse in Antiquity, Stuttgart 2015, 305-346.

H. Braun, Procopius Caesariensis quatenus imitatus sit Thucydidem, Erlangae 1885 (diss.).

L. Brubaker, Sex, Lies, and Intertextuality: The «Secret History» of Prokopios and the Rhetoric of Gender in Sixth-century Byzantium, in L. Brubaker J.H.M. Smith (eds.), Gender in Early Medieval World, East and West, 300-900, Cambridge 2004, 83-101.

A. Cameron, Procopius and the Sixth Century, London New York 1985.

B. Croke, Procopius' «Secret History»: Rethinking the Date, GRBS 45 (2005), 405-431.

G. Greatrex, Perceptions of Procopius in Recent Scholarship, Histos 8 (2014), 76-121.

G. Greatrex, Perceptions of Procopius in Recent Scholarship («Addenda»), Histos 8 (2014), 121a-e.

R. Hirzel, Zur Charakteristik Theopomps, RbM 47 (1892), 359-389.

A. Kaldellis, Procopius of Caesarea: Tyranny, History, and Philosophy at the End of Antiquity, Philadelphia 2004. 
Kaldellis 2009

Kaldellis 2010

Leppin - Meier 2005

Lieberich 1900

Parmeggiani 2003

Parmeggiani 2011

Rubin 1957

Vattuone 2014
A. Kaldellis, The Date and Structure of Prokopios' «Secret History» and His Projected Work on Church History, GRBS 49 (2009), 585-616.

Prokopios, The Secret History with Related Texts, edited and translated, with an introduction, by A. Kaldellis, Indianapolis - Cambridge 2010.

Prokop, Anekdota. Geheimgeschichte des Kaiserhofs von Bysanz, übersetzt und herausgegeben von O. Veh, mit Erläuterungen, einer Einführung und Literaturhinweisen von M. Meier und H. Leppin, Düsseldorf - Zürich 2005.

H. Lieberich, Studien zu den Proömien in der griechischen und byzantinischen Geschichtsschreibung, II, München 1900.

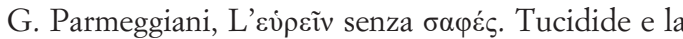
conoscenza del passato, AncSoc 33 (2003), 235-283.

G. Parmeggiani, Eforo di Cuma. Studi di storiografia greca, Bologna 2011.

B. Rubin, s.v. Prokopios (21), in RE XXIII.1, 1957, 273-599.

R. Vattuone, Looking for the Invisible: Theopompus and the Roots of Historiography, in G. Parmeggiani (ed.), Between Thucydides and Polybius: The Golden Age of Greek Historiography, Washington (DC) 2014, 7-37. 